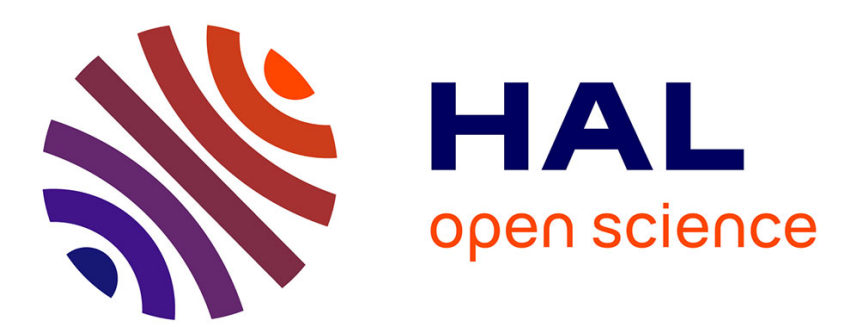

\title{
Automating Cataloging and Discovery of Services for Service-Oriented Robotic Systems
}

\author{
Lucas Bueno Ruas de Oliveira, Diogo Martins, Felipe Amaral, Flavio \\ Oquendo, Elisa Yumi Nakagawa
}

\section{To cite this version:}

Lucas Bueno Ruas de Oliveira, Diogo Martins, Felipe Amaral, Flavio Oquendo, Elisa Yumi Nakagawa. Automating Cataloging and Discovery of Services for Service-Oriented Robotic Systems. Doctoral. 11th Latin American Robotics Symposium (LARS), São Carlos, Brazil. 2014, pp.20. cel-01113218

\section{HAL Id: cel-01113218 \\ https://hal.science/cel-01113218}

Submitted on 4 Feb 2015

HAL is a multi-disciplinary open access archive for the deposit and dissemination of scientific research documents, whether they are published or not. The documents may come from teaching and research institutions in France or abroad, or from public or private research centers.
L'archive ouverte pluridisciplinaire HAL, est destinée au dépôt et à la diffusion de documents scientifiques de niveau recherche, publiés ou non, émanant des établissements d'enseignement et de recherche français ou étrangers, des laboratoires publics ou privés. 


\section{Automating Cataloging and Discovery of Services for Service-Oriented Robotic Systems}

Lucas Bueno R. Oliveira ${ }^{1,2}$, Diogo B. Martins ${ }^{1}$, Felipe A. Amaral ${ }^{1}$, Flavio Oquendo ${ }^{2}$, and Elisa Yumi Nakagawa ${ }^{1}$ buenolro@icmc.usp.br, dbrdem@usp.br, felipeaa@usp.br, flavio.oquendo@irisa.fr, and elisa@icmc.usp.br

${ }^{1}$ Dept. of Computer Systems, University of São Paulo - USP, São Carlos, Brazil

${ }^{2}$ IRISA Research Institute University of South Brittany, Vannes, France

LARS 2014, São Carlos/SP

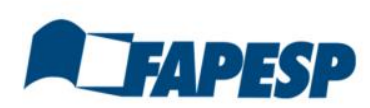




\section{Agenda}

Introduction

Developing RoboSeT

Case Study

Discussion

Conclusion and Future Work 


\section{Introduction}

- Robots have been used in several areas of the society

- Complexity and diversity challenge the development of robotic systems

- Service-Oriented Architecture (SOA) promotes better reusability and flexibility to robotic systems

- Several Service-Oriented Robotic Systems (SORS) can be found in the literature

- Development environments for SORS are also available 


\section{Introduction}

- Motivation:

- SORS development environments do not provide facilities for location and selection of services

Documentation
fuerte groovy hydro




\section{Introduction}

- Motivation:

- SORS development environments do not provide facilities for location and selection of services

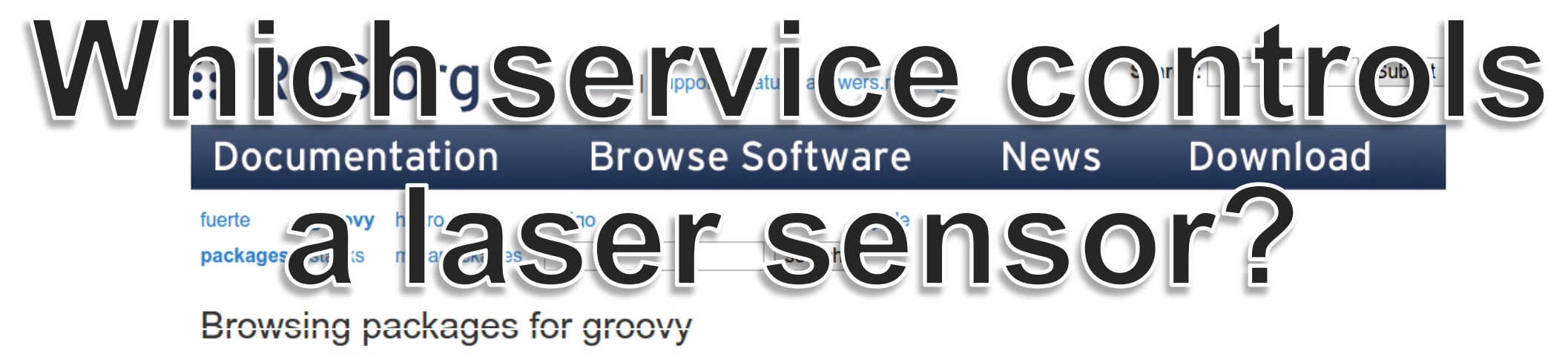

\begin{tabular}{|c|c|c|}
\hline Name & Maintainers / Authors & Description \\
\hline ADEPT_VIPER_S650_arm_navigation & Shaun Edwards & ADEPT_VIPER_S650_arm_navigation \\
\hline ADEPT_VIPER_S650_ikfast_arm_navigation & Jorge Nicho & ADEPT_VIPER_S650_arm_navigation \\
\hline AllegroHand & $\begin{array}{l}\text { Alex Alspach (SimLab), Seungsu Kim } \\
\text { (EPFL) }\end{array}$ & AllegroHand \\
\hline AllegroHand_keyboard & Alex & AllegroHand_keyboard \\
\hline CANOpen_driver & Tim Fröhlich & CANOpen_driver \\
\hline LMS1xx & Konrad Banachowicz & Driver for SICK LMS1xx. \\
\hline
\end{tabular}




\section{Introduction}

- Motivation:

- SORS development environments do not provide facilities for location and selection of services

Isothis service the

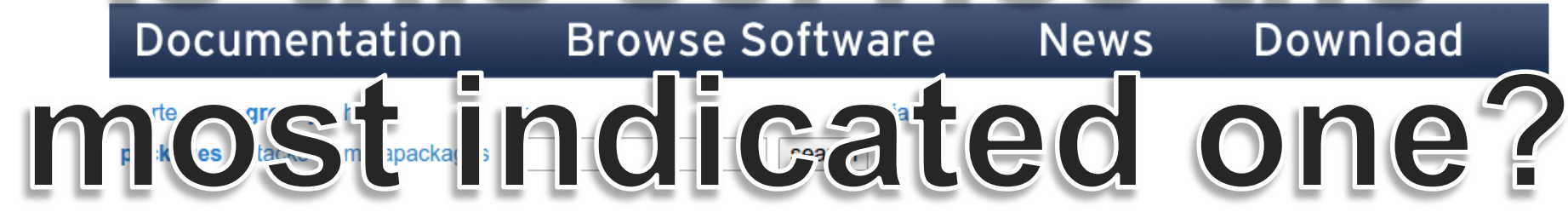

Browsing packages for groovy

\begin{tabular}{|c|c|c|}
\hline Name & Maintainers / Authors & Description \\
\hline ADEPT_VIPER_S650_arm_navigation & Shaun Edwards & ADEPT_VIPER_S650_arm_navigation \\
\hline ADEPT_VIPER_S650_ikfast_arm_navigation & Jorge Nicho & ADEPT_VIPER_S650_arm_navigation \\
\hline AllegroHand & $\begin{array}{l}\text { Alex Alspach (SimLab), Seungsu Kim } \\
\text { (EPFL) }\end{array}$ & AllegroHand \\
\hline AllegroHand_keyboard & Alex & AllegroHand_keyboard \\
\hline CANOpen_driver & Tim Fröhlich & CANOpen_driver \\
\hline LMS1xx & Konrad Banachowicz & Driver for SICK LMS1xx. \\
\hline
\end{tabular}




\section{Introduction}

- Main goal:

- To present RoboSeT (Robotics Services Semantic Search (ool), a mechanism that supports cataloging and discovery of services for robotic systems using semantic information. 


\section{Developing RoboSeT}

- First step: Establishment of a common vocabulary [1]

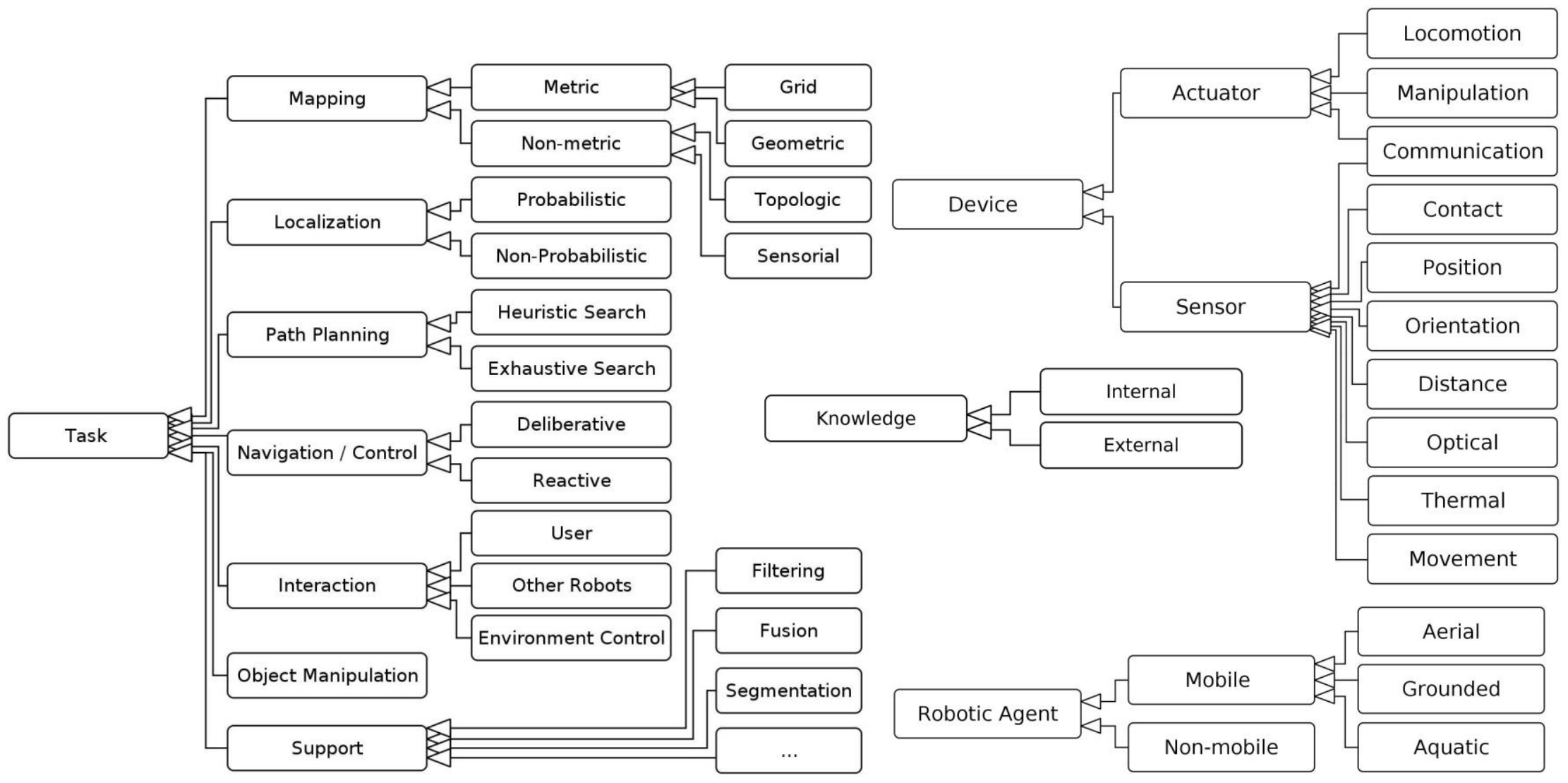

[1] Oliveira, L. B. R.; Osório, F. S.; Oquendo, F.; Nakagawa, E. Y.; Towards a Taxonomy of Services for Developing ServiceOriented Robotic Systems. In: SEKE'14, 2014, Vancouver, Canada, p. 344-349. 


\section{Developing RoboSeT}

- RoboSeT is divided into two main subsystems:

- Service repository (Web Interface)

- Service plug-ins (GUI or command line)

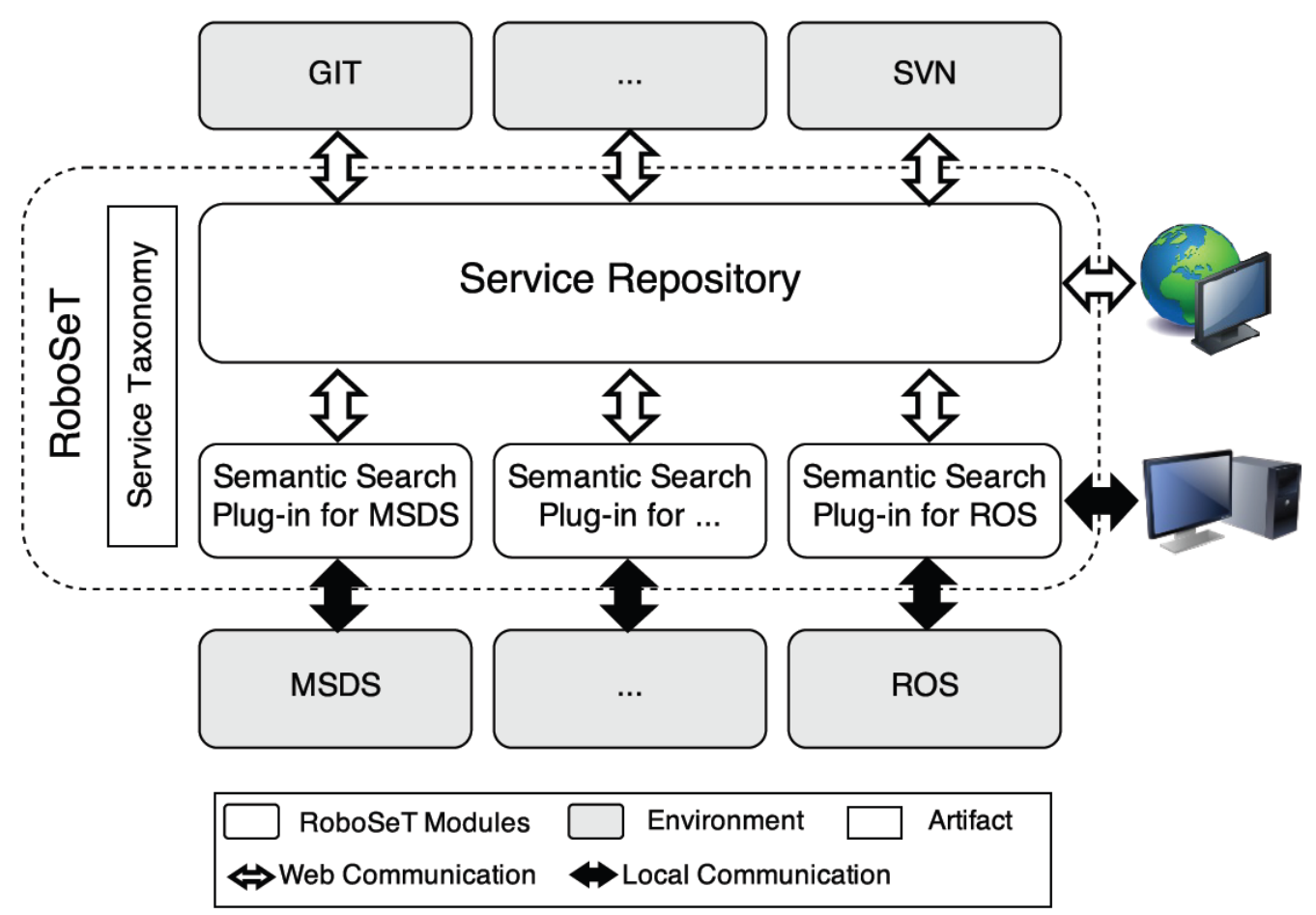




\section{Developing RoboSeT}

- Service repository:

- Account management

- Service publication

- Service search and detailing

- Service management

- News about services

- Plug-ins:

- Service search

- Service identification

- Service obtaining and deployment

- Feedback about services (quality, bugs, comments) 


\section{Case study}

- Design of a robotic system for robust navigation

- Motion Planning

- Trajectory generation

- Obstacle detection and representation

- Obstacle avoidance

- Position and velocity control

- Localization

- Laser controller

- Pioneer P3-DX controller 


\section{Case study}

- Mapping requirements into service types

\begin{tabular}{ll}
\hline Functionality & Service type \\
\hline Motion Planning & Service/Task/Path planning \\
\hline Trajectory generation & Service/Task/Path planning \\
\hline Obstacle detection and representation & Service/Task/Mapping \\
\hline Obstacle avoidance & Service/Task/Path planning \\
\hline Position and velocity control & Service/Task/Navigation \\
\hline Localization & Service/Task/Localization \\
\hline Encoder controller & Service/Device/Sensor/Movement \\
\hline Differential drive controller & Service/Device/Actuator/Locomotion \\
Laser controller & Service/Device/Sensor/Distance \\
\hline
\end{tabular}




\section{Case study}

- Searching for ROS services

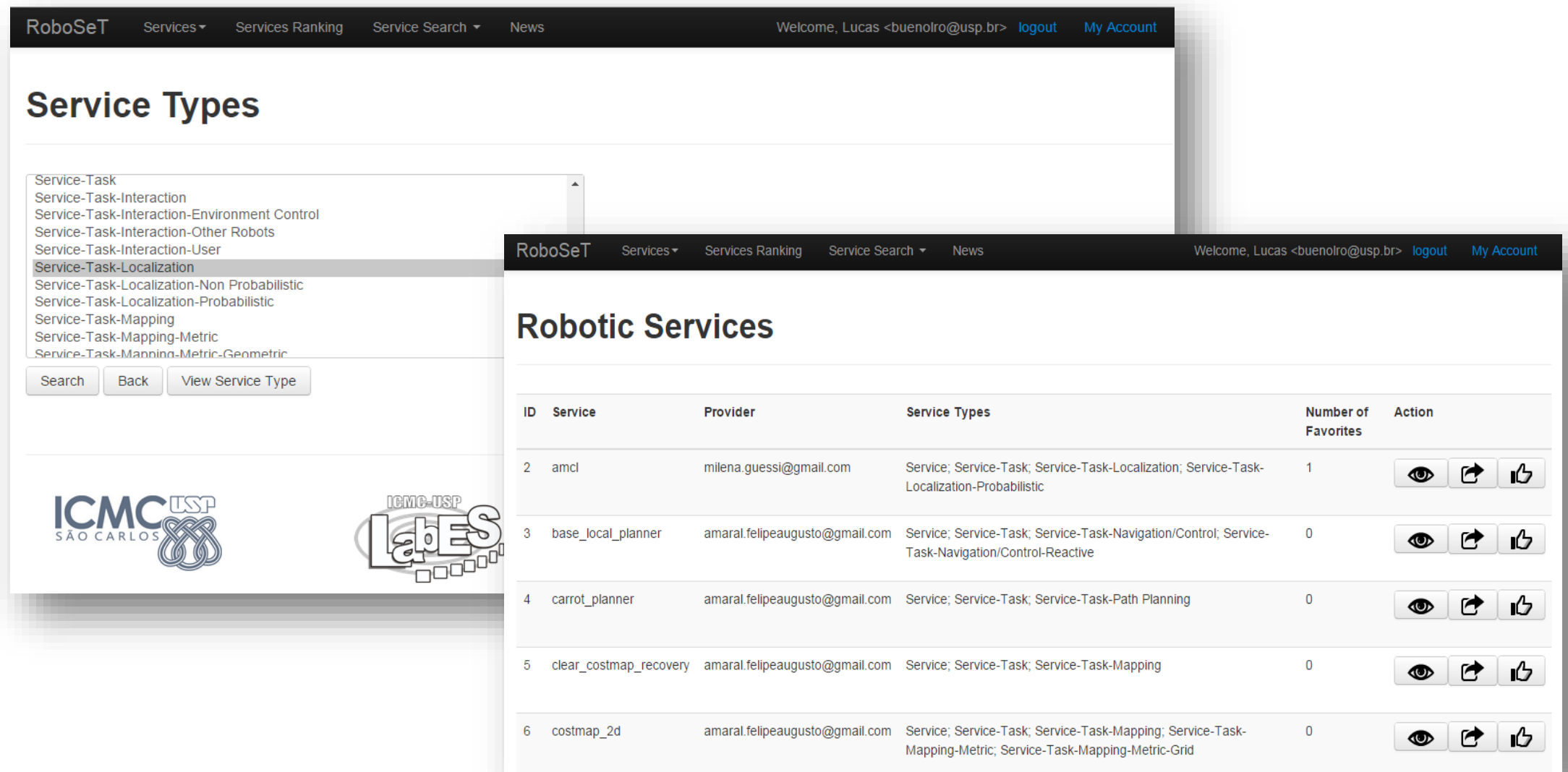




\section{Case study}

- Searching for ROS services (Part I)

\begin{tabular}{|c|c|c|}
\hline Functionality & Task service type & ROS Service \\
\hline Motion Planning & $\begin{array}{l}\text { Path planning/Heuristic } \\
\text { Search }\end{array}$ & $\begin{array}{l}\text { NavfnROS, } \\
\text { CarrotPlanner }\end{array}$ \\
\hline Trajectory generation & $\begin{array}{l}\text { Path planning/Heuristic } \\
\text { Search }\end{array}$ & $\begin{array}{l}\text { TrajectoryPlannerROS, } \\
\text { DWAPlannerROS }\end{array}$ \\
\hline $\begin{array}{l}\text { Obstacle detection and } \\
\text { representation }\end{array}$ & Mapping/Metric/Grid & CostMap2D \\
\hline Obstacle avoidance & $\begin{array}{l}\text { Path planning/Heuristic } \\
\text { Search }\end{array}$ & $\begin{array}{l}\text { TrajectoryPlannerROS, } \\
\text { DWAPlannerROS }\end{array}$ \\
\hline $\begin{array}{l}\text { Position and velocity } \\
\text { control }\end{array}$ & Navigation & MoveBase \\
\hline Localization & Localization/Probabilistic & $\mathrm{Amcl}$ \\
\hline
\end{tabular}




\section{Case study}

- Searching for ROS services (Part II)

\begin{tabular}{lll}
\hline Functionality & Device service type & ROS Service \\
\hline Encoder controller & Sensor/Movement & RosAria \\
$\begin{array}{l}\text { Differential drive } \\
\text { controller }\end{array}$ & Actuator/Locomotion & RosAria \\
Laser controller & Sensor/Distance & SICK Toolbox \\
\hline
\end{tabular}




\section{Case study}

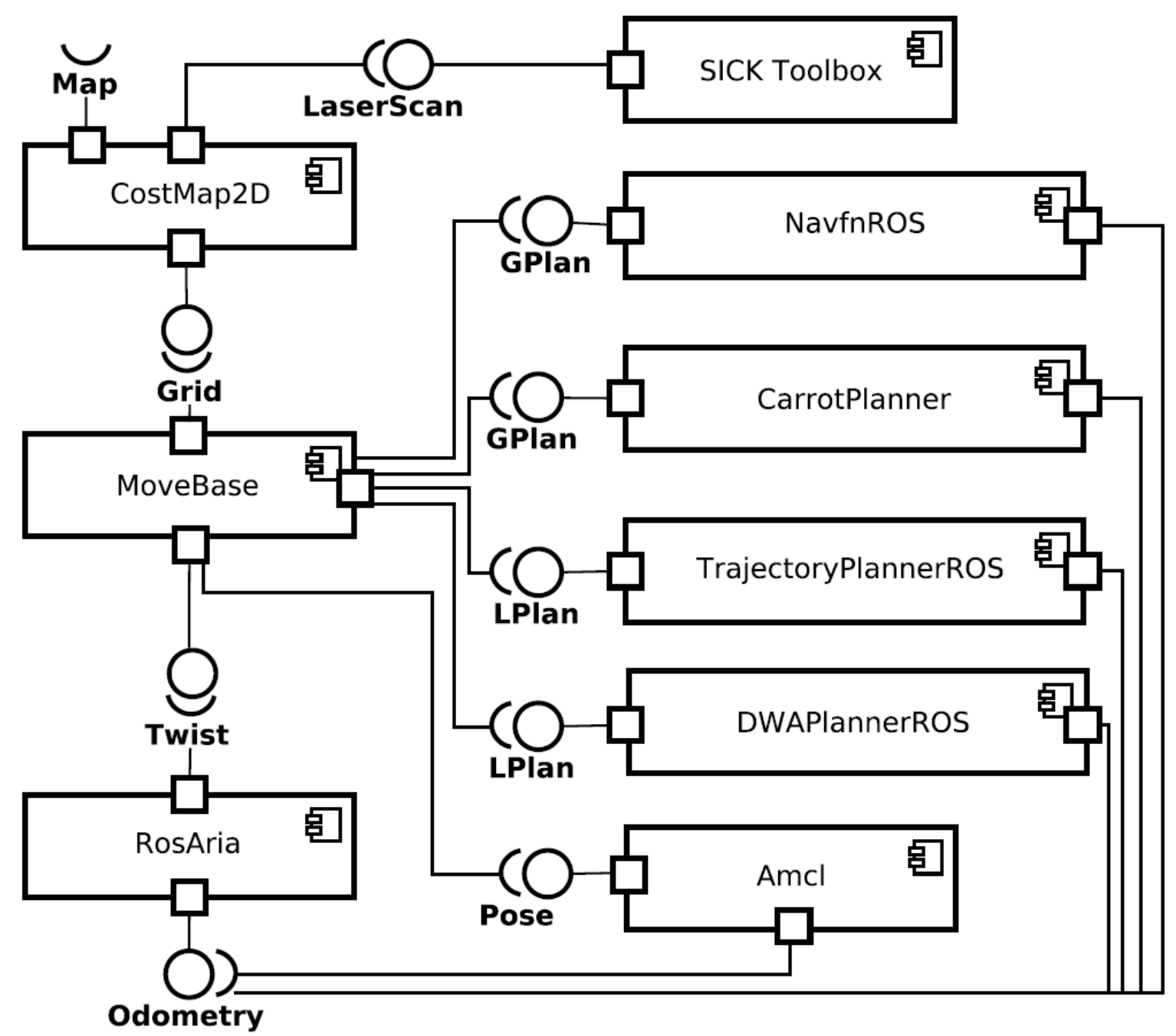




\section{Discussion}

- Advantages:

- Services can be transparently shared and discovered

- Services that are easier to be found are more likely to be reused

- Structured information can support identification of more suitable services

- Reuse improvements positively influence productivity in software systems development

- Community feedback can guide the development of services of better quality 


\section{Discussion}

- Main limitation:

- RoboSeT is depended on community adoption and cooperation

- Current initiatives to mitigate such limitation:

- To promote RoboSeT in the robotics community

- To release an open source version of RoboSeT 


\section{Conclusion and Future Work}

- SOA is a promising architectural style for robotics

- A mechanism for supporting cataloging, publishing, and discovery of services can contribute to the SORS development

- Results indicate that RoboSeT can ease the discovery of services for SORS

- Future work:

- To perform an experiment

- To develop new functionalities and plug-ins 


\section{Automating Cataloging and Discovery of Services for Service-Oriented Robotic Systems}

Lucas Bueno R. Oliveira ${ }^{1,2}$, Diogo B. Martins ${ }^{1}$, Felipe A. Amaral ${ }^{1}$, Flavio Oquendo ${ }^{2}$, and Elisa Yumi Nakagawa ${ }^{1}$ buenolro@icmc.usp.br, dbrdem@usp.br, felipeaa@usp.br, flavio.oquendo@irisa.fr, and elisa@icmc.usp.br

${ }^{1}$ Dept. of Computer Systems, University of São Paulo - USP, São Carlos, Brazil

${ }^{2}$ IRISA Research Institute University of South Brittany, Vannes, France

LARS 2014, São Carlos/SP

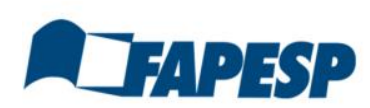

